\title{
TECHNIQUES FOR PREPARING RICCIAS FOR HERBARIUM STUDY
}

\author{
Michael R. Mayfield, Marie C. Cole and Warren H. Wagner, Jr. ${ }^{1}$
}

During investigations of the hepatic family Ricciaceae, the poor quality of most existing herbarium specimens of this group came into question. Herbarium specimens of good quality serve a dual purpose, being necessary as biogeographic records and taxonomic standards. The thalli of the Ricciaceae are delicate, and failure to remove soil particles from their rhizoids results in the gradual breakage and deterioration of specimens. With time, dried soil particles dislodge from the rhizoids and thallus surfaces. As the packets containing specimens are moved, the mechanical action of the particles destroy the thalli. The majority of specimens we studied were too damaged for taxonomic study or verification.

Having recognized a need for quality herbarium specimens in our own studies, we have developed an effective and efficient process for the removal of soil from freshly collected Ricciaceae. Following this cleaning process, the specimens are mounted in two ways. The first involves lightly pressing the plants, then pasting them to $3 \times 5^{\prime \prime}$ cards for placement in herbarium packets. Specimens prepared in this manner are thus suitable for routine herbarium work. A second method involves alcohol dehydration of the thalli in preparation for permanent slide mounts. Specimens prepared in this way serve as more critical taxonomical and morphological standards and can also be used as teaching materials.

\section{Method 1}

Freshly collected specimens are carefully lifted from the substrate with the edge of a razor blade. Approximately $1 / 8$ inch of substrate may be retained to facilitate cleaning without initial damage to the thalli or rhizoids. A screen consisting of fine mesh cloth (mosquito netting or bridal veil work well) tautly stretched in an embroidery hoop is an effective cleaning tool. The thalli are positioned on the screen, a second cleaning screen is placed over the plants. This secures the plants while a stream of water washes soil from the rhizoids. The force of the water is critical. Too strong a stream will damage the thalli; a weak or undirected stream will fail to remove adherent particles. An efficient, directed stream of water may be obtained by slipping pliable rubber tubing over an eyedropper. A narrow gauge piece of wire secures the tubing to the dropper. This simple tubing plus dropper set-up may be attached to a tapered laboratory faucet. Moderate care in washing the specimens will produce clean ventral surfaces.

After the specimens are satisfactorily cleaned, they should be lightly pressed. We find old telephone books to be convenient for this purpose. The specimens, transferred to labelled slips of paper, are placed in folded wax paper. The wax paper is put between the pages of the phonebook. Using water-soluble glue, the dried specimens are mounted on $3 \times 5^{\prime \prime}$ cards for placement in herbarium packets. As the specimens are freed from their destructive soil particles, they remain intact within the packets. The specimens may be later rehydrated with boiling water for taxonomic studies.

We have used this technique to process the majority of specimens that we have collected.

1 Division of Biological Sciences and Herbarium, University of Michigan, Ann Arbor, MI 48109, U.S.A. We acknowledge the help and suggestion of Howard A. Crum. 
The time required for processing is minimal and 30-50 thalli fit on a medium-sized hoop for efficient cleaning.

\section{Method 2}

When higher quality specimens are required for more detailed study, this method should be employed. The soil-cleansing process is similar to that of Method 1, but periodic examination with a dissecting microscope is necessary to obtain as clean a specimen as possible. Following the wash, the plants are run through an alcohol dehydration series using $70 \%, 90 \%$ and $100 \%$ ETOH. The dehydrated specimens are then placed into a $1: 1$ ethyl alcohol : xylene solution before transfer to $100 \%$ xylene. Remaining soil particles that have dislodged during dehydration should be removed with fine needles. The prepared thalli are mounted on glass slides using a xylene soluble mounting medium. Since the plants are thick and porous, small weights placed on the coverslips prevent the accumulation of air bubbles. The time required to clean and prepare the specimens using Method 2 is greater than that of the previous method, but results in higher quality specimens.

The materials required for cleaning and mounting Ricciaceae according to methods outlined in this paper are available in most laboratories. Both methods yield excellent reference specimens. We have also employed these same techniques in successfully cleaning and mounting hornworts and other thallose liverworts as well. 